\title{
Wavelet-based medical image fusion via a non-linear operator
}

\begin{abstract}
Medical image fusion has been extensively used to aid medical diagnosis by combining images of various modalities such as Computed Tomography (CT) and Magnetic Resonance Image (MRI) into a single output image that contains salient features from both inputs. This paper proposes a novel fusion algorithm through the use of a non-linear fusion operator, based on the low sub-band coefficients of the Discrete Wavelet Transform (DWT). Rather than employing the conventional mean rule for approximation sub-bands, a modified approach is taken by the introduction of a non-linear fusion rule that exploits the multimodal nature of the image inputs by prioritizing the stronger coefficients. Performance evaluation of CT-MRI image fusion datasets based on a range of wavelet filter banks shows that the algorithm boasts improved scores of up to $92 \%$ as compared to established methods. Overall, the non-linear fusion rule holds strong potential to help improve image fusion applications in medicine and indeed other fields.
\end{abstract}

Keyword: Medical image fusion; Non-linear fusion operator; Wavelet transforms; Medical imaging 